\title{
Multi-Keyhole MIMO Channels: Asymptotic Analysis of Outage Capacity
}

\author{
George Levin and Sergey Loyka \\ School of Information Technology and Engineering (SITE) \\ University of Ottawa \\ 161 Louis Pasteur, Ottawa, Ontario, Canada, K1N 6N5 \\ Tel: (613) 562-5800 ext. 2198, Fax: (613) 562-5175 \\ Email: \{glevin, sloyka\}@site.uottawa.ca
}

\begin{abstract}
Keyhole MIMO channels were predicted theoretically and also observed experimentally. However, they are not often encountered in practice since the assumption of a single propagation eigenmode is only a rough approximation of real propagation environments. To overcome this problem, the paper extends the single-keyhole channel model by introducing a "multi-keyhole channel", which includes a number of statistically independent keyholes. Correlated full-rank and rank-deficient multi-keyhole channels are considered in detail. It is shown that under some general conditions the full-rank multikeyhole channel is asymptotically Rayleigh fading, if the number of keyholes is large. When the number of both $T x$ and $R x$ antennas is large, the asymptotic capacity of a rank-deficient multi-keyhole channel is a sum of the capacities of the equivalent single-keyhole channels. The outage capacity distribution of both full-rank and rank-deficient multi-keyhole channels is asymptotically Gaussian. Based on the asymptotic capacity analysis, full ordering scalar measure of MIMO channel correlation and power imbalance is introduced.
\end{abstract}

Index Terms - MIMO system, keyhole channel, outage capacity, correlation.

\section{INTRODUCTION}

Multiple-Input-Multiple-Output (MIMO) channels have gained significant interest in the last decade due to high spectral efficiency. One of the major statistical characteristics of MIMO fading channels is their outage capacity, which gives an ultimate upper limit on the error-free information rate with a given probability of outage [1]. The outage capacity distribution of various channels has been extensively studied, and many analytical and empirical results have been obtained. For example, closed-form expressions for the outage capacity distribution of non-correlated and semicorrelated Rayleigh MIMO channels have been found [2]. Chizhik et al [3] analytically predicted a keyhole channel, which can be modeled as a cascade of two Rayleigh fading channels separated by a single keyhole whose dimensions are much smaller than the wavelength. The presence of the keyhole degenerates the channel, i.e. its rank is one regardless the number of $\mathrm{Tx}$ and Rx antennas [3]. Consequently, the capacity of such channels deteriorates significantly comparing to the Rayeigh channel with the same number of $\mathrm{Tx}$ and Rx antennas, even though the channel matrix entries are uncorrelated. Outage capacity distribution of singlekeyhole channels is studied in [4]. Closed-form expressions for the mean (ergodic) capacity of a spatially uncorrelated single-keyhole channel are obtained in [5]. Even though the single-keyhole channels may appear in some propagation scenarios, they are not often encountered in practice as the assumption of a single propagation eigenmode is only a rough approximation of real propagation scenarios [6]. Motivated by recent studies of the single-keyhole channel [4], we introduce a multi-keyhole channel to generalize and expand the range of applicability of the keyhole channel model. While the statistics of the keyhole channel is significantly different from the Rayleigh one, we show that under some general conditions the Rayleigh channel is a limiting case of a full-rank multi-keyhole channel when the number of keyholes is large. This establishes a link between keyhole and Rayleigh channels. The outage capacity of the Rayleigh channel upperbounds that of the multi-keyhole one. Moreover, the full rank multi-keyhole model provides a motivation for the Kronecker correlation model [7]. When the number of antennas is large, the capacity of the rank-deficient multi-keyhole channel is a sum of the capacities of the equivalent single-keyhole channels. While the mean capacity of such a channel increases with the number of keyholes, its outage capacity may decrease. Moreover, the outage capacity distribution of both full-rank and rank-deficient multi-keyhole channels is asymptotically Gaussian. This fact may indicate that Gaussian distribution has a certain degree of universality for outage capacity analysis of MIMO channels in general.

Motivated by the results above, we introduce a generic scalar measure of channel correlation and power imbalance in terms of their impact on the channel capacity. The significant advantage of this measure, as compared to that based on the majorization theory [8], is that any two channel can be compared without exceptions. Using this measure, we show analytically that both the correlation and the power imbalance have a negative impact on the asymptotic outage capacity.

\section{Single-Keyhole MiMO Channel CAPacity}

Consider a spatially correlated single-keyhole MIMO channel with $n_{t}$ Tx and $n_{r}$ Rx antennas (see Fig. 1). Let the element $H_{k m}, k=1 . . n_{r} ; m=1 \ldots n_{t}$, of the channel transfer matrix $\mathbf{H}$ be a complex channel gain from the $m$-th transmit to the $k$-th receive antenna. The gain matrix of the keyhole channel is given by [3]

$$
\mathbf{H}=\mathbf{h}_{r} \mathbf{h}_{t}^{H}
$$




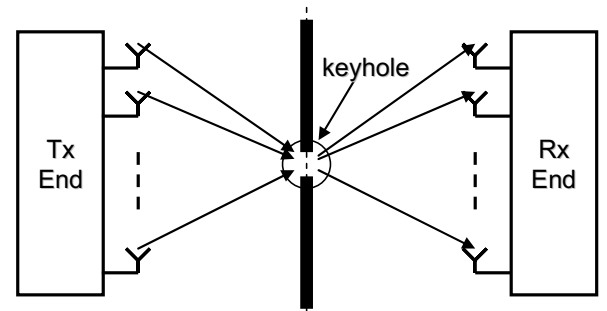

Fig. 1. A keyhole MIMO channel. Each end has rich multipath so that the sub-channels are correlated Rayleigh fading.

where $(\cdot)^{H}$ denotes the Hermitian transpose, $\mathbf{h}_{t}\left[n_{t} \times 1\right]$ and $\mathbf{h}_{r}\left[n_{r} \times 1\right]$ are mutually independent random vectors representing the complex gains from the transmit antennas to the keyhole and from the keyhole to the receive antennas respectively. Assuming that the keyhole channel is a cascade of two correlated Rayleigh fading channels, $\mathbf{h}_{t}$ and $\mathbf{h}_{r}$ are complex circular symmetric correlated Gaussian vectors with correlation matrices $\mathbf{R}_{t}=E\left\{\mathbf{h}_{t} \mathbf{h}_{t}^{H}\right\}$ and $\mathbf{R}_{r}=E\left\{\mathbf{h}_{r} \mathbf{h}_{r}^{H}\right\}$ respectively, where $E\{\cdot\}$ denotes expectation. $\mathbf{H}$ is normalized so that $E\left\{\|\mathbf{H}\|^{2}\right\}=n_{t} n_{r}$, where \|\| is the $L_{2}$ norm, and $n_{t}^{-1} E\left\{\left\|\mathbf{h}_{t}\right\|^{2}\right\}=n_{r}^{-1} E\left\{\left\|\mathbf{h}_{r}\right\|^{2}\right\}=1$, which also implies $n_{t}^{-1}$ trace $\left\{\mathbf{R}_{t}\right\}=n_{r}^{-1}$ trace $\left\{\mathbf{R}_{r}\right\}=1$.

From [1], when the channel state information (CSI) is available at the $\mathrm{Rx}$ but not the $\mathrm{Tx}$ end, the instantaneous capacity (i.e. the capacity for a given channel realization) of a quasi-static frequency flat MIMO channel in natural units [nat] is given by:

$$
C=\ln \left(\operatorname{det}\left[\mathbf{I}+\gamma_{0} \mathbf{H} \mathbf{H}^{H} / n_{t}\right]\right)
$$

where det is the determinant, $\mathbf{I}$ is $\left[n_{r} \times n_{r}\right]$ identity matrix and $\gamma_{0}$ is the average SNR per Rx antenna. The exact expression for the cumulative distribution functions (CDF) of $C$ (the outage capacity distribution) when $\mathbf{R}_{t}$ or $\mathbf{R}_{r}$ are non-singular and have distinct eigenvalues has been obtained in [4]. However, this expression is not explicit in parameters and does not allow for significant insight. To obtain such insight, we resort to an asymptotic analysis, when both $n_{t}$ and $n_{r}$ are large.

\section{Asymptotic OUtage CAPACITY Distribution OF A SINGLE-KEYHOLE MIMO CHANNEL}

Here, we briefly summarize the main results in [4] starting with the following theorem:

Theorem 1: Let $C$ be an instantaneous capacity of the correlated single-keyhole channel. When both $n_{t}$ and $n_{r}$ tend to infinity, the distribution of $C$ is Gaussian in probability if $n_{t}^{-1}$ trace $\left\{\mathbf{R}_{t}\right\}<\infty, \quad n_{t}^{-2}\left\|\mathbf{R}_{t}\right\|^{2} \rightarrow 0 \quad$ as $\quad n_{t} \rightarrow \infty, \quad$ and $n_{r}^{-1}$ trace $\left\{\mathbf{R}_{r}\right\}<\infty, n_{r}^{-2}\left\|\mathbf{R}_{r}\right\|^{2} \rightarrow 0$ as $n_{r} \rightarrow \infty$. Moreover, if the channel is normalized so that $n_{t}^{-1}$ trace $\left\{\mathbf{R}_{t}\right\}=1$ and $n_{r}^{-1}$ trace $\left\{\mathbf{R}_{r}\right\}=1$, the mean $\mu$ and the variance $\sigma^{2}$ of $C$ are as follows:

$$
\mu=\ln \left(1+n_{r} \gamma_{0}\right) ; \quad \sigma^{2}=n_{t}^{-2}\left\|\mathbf{R}_{t}\right\|^{2}+n_{r}^{-2}\left\|\mathbf{R}_{r}\right\|^{2}
$$

A proof is given in [4]. The conditions of Theorem 1 apply for both uncorrelated and correlated single-keyhole channels. Hence, the outage capacity distribution of an uncorrelated single-keyhole MIMO channel is asymptotically Gaussian with the mean as in (3) and the variance $\sigma^{2}=n_{t}^{-1}+n_{r}^{-1}$. The following corollary follows immediately from Theorem 1 .

Corollary 1: Asymptotically, the channel correlation enters into the outage capacity distribution through the norm only, i.e. even though two correlation matrices $\mathbf{R}_{1}$ and $\mathbf{R}_{2}$ (at either end) are different, they affect the capacity in the same way if $\left\|\mathbf{R}_{1}\right\|=\left\|\mathbf{R}_{2}\right\|$.

Following (3), it is not always true that an increase in the number of antennas decreases the variance and hence the outage probability, but only if $\sigma^{2}$ is monotonically decreasing with $n_{t}$ and $n_{r}$, i.e. $\left\|\mathbf{R}_{t}\right\|$ and $\left\|\mathbf{R}_{r}\right\|$ increase not faster than $n_{t}{ }^{1-\varepsilon_{1}}$ and $n_{r}{ }^{1-\varepsilon_{2}}$ respectively for some $\varepsilon_{1}, \varepsilon_{2}>0$.

Since $\mu$ in (3) is a function of $n_{r}$ and $\gamma_{0}$ but not $\mathbf{R}$ (either $\mathbf{R}_{t}$ or $\mathbf{R}_{r}$ ), the immediate conclusion is that the correlation has no effect on the asymptotic mean capacity, but only on the variance. In contrast, increase in the average SNR increases the mean capacity while the variance remains unchanged. Note that the asymptotic mean capacity in (3) and the upper bound on the mean capacity of the finite order single-keyhole channel proposed in [5] are identical, i.e. (3) shows that the bound is asymptotically tight.

To analyze the effect of $\sigma^{2}$ in (3), consider two singlekeyhole channels with the same $\gamma_{0}, n_{t}$ and $n_{r}$, but different $\mathbf{R}$, either $\mathbf{R}_{t}$ or $\mathbf{R}_{r}$. Under the conditions of Theorem 1, the outage capacity distribution of both channels is asymptotically Gaussian with equal means $\mu_{1}=\mu_{2}=\mu$ and variances $\sigma_{1}^{2}, \sigma_{2}^{2}$. Without loss of generality, assume that $\sigma_{1}^{2}>\sigma_{2}^{2}$. Compare two equal-mean Gaussian CDFs $F_{C 1}(x)$ (with variance $\sigma_{1}^{2}$ ) and $F_{C 2}(x)$ (with variance $\sigma_{2}^{2}$ ). Apparently, they have a cross point at $x_{0}=\mu$ such that $F_{C 1}\left(x_{0}\right)=F_{C 2}\left(x_{0}\right)=0.5$. For $x<x_{0}, F_{C 1}(x)>F_{C 2}(x)$ and for $x>x_{0}, F_{C 1}(x)<F_{C 2}(x)$, i.e. at outage probabilities less than 0.5 , the keyhole channel with higher $\sigma^{2}$ has smaller outage capacity. The opposite is true at outage probabilities higher than 0.5 , however this range of outage probabilities has little importance from the practical point of view.

\section{SCALAR MEASURES OF CORRELATION AND POWER IMBALANCE}

Let $\mathbf{R}$ (either $\mathbf{R}_{t}$ or $\mathbf{R}_{r}$ ) belongs to $\mathfrak{R}$, where $\mathfrak{R}$ is a set of all $n \times n$ correlation matrices such that $\operatorname{trace}(\mathbf{R})=n$. It is straightforward to show using the Cauchy-Schwarz inequality that

$$
n^{-2}\|\mathbf{R}\|^{2} \geq n^{-1},
$$

with the equality if all $\lambda_{k}=1, k=1 \ldots n$, where $\lambda_{k}$ are eigenvalues of $\mathbf{R}$ (i.e. if $\mathbf{R}=\mathbf{I}$ ). Thus, $n^{-2}\|\mathbf{R}\|^{2}$ achieves the minimum when the channel at the $\operatorname{Tx}(\operatorname{Rx})$ end is uncorrelated with the same power at each $\operatorname{Tx}(\mathrm{Rx})$ antenna. Furthermore, since every $\mathbf{R} \in \mathfrak{R}$ is positive semi-definite $\left(\lambda_{k} \geq 0\right)$, it is easy to show that 


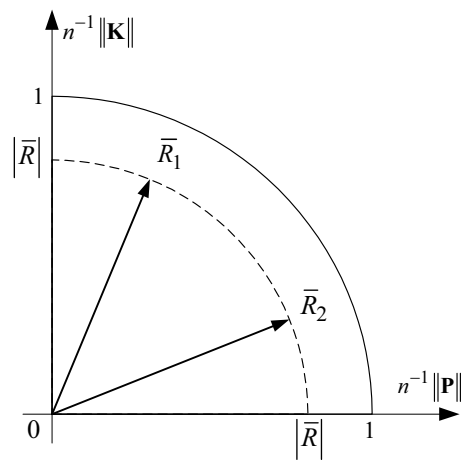

Fig. 2. Geometrical interpretation of the power imbalance and correlation effects.

$$
n^{-2}\|\mathbf{R}\|^{2} \leq 1,
$$

with the equality if $\lambda_{k}=n$ for some $k$, and $\lambda_{m}=0$ $\forall m \neq k$. Thus, $n^{-2}\|\mathbf{R}\|^{2^{k}}$ achieves the maximum when the channel at $\operatorname{Tx}(\operatorname{Rx})$ end is fully correlated. Based on (4) and (5), there are two major effects that can increase $n^{-2}\|\mathbf{R}\|^{2}$ : (i) non-uniform power distribution across the antennas (also termed power imbalance) and (ii) non-zero correlation. To analyze those effects separately, let us split $\mathbf{R} \in \mathfrak{R}$ into a sum of two matrices as follows:

$$
\mathbf{R}=\mathbf{K}+\mathbf{P}
$$

where $\mathbf{P}=\operatorname{diag}\{\mathbf{R}\}-\mathbf{I}$ and $\mathbf{K}=\mathbf{R}-\mathbf{P} ; \operatorname{diag}\{\mathbf{R}\}$ is the diagonal matrix whose main diagonal is that of $\mathbf{R}$. Clearly, $\mathbf{P}$ and $\mathbf{K}$ account for the power imbalance and the correlation respectively. As for any $\mathbf{R} \in \mathfrak{R}, \operatorname{trace}(\mathbf{K})=n$ and $\operatorname{trace}(\mathbf{P})=0$, it is straightforward to show that the decomposition (6) is norm-orthogonal, i.e.

$$
n^{-2}\|\mathbf{R}\|^{2}=n^{-2}\|\mathbf{K}\|^{2}+n^{-2}\|\mathbf{P}\|^{2}
$$

Moreover, it can be shown that

$$
0 \leq n^{-2}\|\mathbf{P}\|^{2} \leq 1-n^{-1}
$$

where the lower bound is achieved when all antennas have the same power (no power imbalance), i.e. $\operatorname{diag}\{\mathbf{R}\}=\mathbf{I}$, and the upper bound is achieved when there is only one effective Tx or Rx antenna, i.e. $R_{k k}=n$ for some $k$, and $R_{m m}=0$ $\forall m \neq k$. Furthermore, since $n^{-2}\|\mathbf{K}\|^{2} \leq n^{-2}\|\mathbf{R}\|^{2}$ (see (7)), and the equality in (4) is achieved only for $n^{-2}\|\mathbf{K}\|^{2}=n^{-2}\|\mathbf{R}\|^{2}$, it follows that

$$
n^{-1} \leq n^{-2}\|\mathbf{K}\|^{2} \leq 1
$$

where the lower bound is achieved when $\mathbf{K}=\mathbf{I}$ (the channel at $\operatorname{Tx}(\operatorname{Rx})$ end is uncorrelated), and the upper bound is achieved when the channel at the $\operatorname{Tx}(\operatorname{Rx})$ end is fully correlated. Motivated by Corollary 1 and the discussion above, we introduce two following definitions.

Definition 1: A channel with correlation matrix $\mathbf{R}_{1} \in \mathfrak{R}$ is said to be equally or more correlated than that with $\mathbf{R}_{2} \in \mathfrak{R}$ if

$$
n^{-2}\left\|\mathbf{K}_{1}\right\|^{2} \geq n^{-2}\left\|\mathbf{K}_{2}\right\|^{2}
$$

where $\mathbf{K}_{1}$ and $\mathbf{K}_{2}$ correspond to $\mathbf{R}_{1}$ and $\mathbf{R}_{2}$ respectively through (6). This scalar measure of the channel correlation is alternative to the measure given in [8], for channels with large $n$. Unlike [8], (10) is not based on the majorization theory and provides a complete (full-ordering) correlation characterization with no exception (i.e. any two $\mathbf{R}_{1}, \mathbf{R}_{2} \in \mathfrak{R}$ can be compared, see also the remark to the Definition 1 in [8]).

Definition 2: A channel with correlation matrix $\mathbf{R}_{1} \in \mathfrak{R}$ has the same or more non-uniform power distribution (power imbalance) than that with $\mathbf{R}_{2} \in \mathfrak{R}$ if

$$
n^{-2}\left\|\mathbf{P}_{1}\right\|^{2} \geq n^{-2}\left\|\mathbf{P}_{2}\right\|^{2}
$$

where $\mathbf{P}_{1}$ and $\mathbf{P}_{2}$ correspond to $\mathbf{R}_{1}$ and $\mathbf{R}_{2}$ respectively through (6). Note that for any $\mathbf{R} \in \mathfrak{R}, n^{-2}\|\mathbf{K}\|^{2} \in(0 ; 1]$ and $n^{-2}\|\mathbf{P}\|^{2} \in[0 ; 1)$ as $n \rightarrow \infty$.

To get some insight, consider a simple geometrical interpretation of Definitions 1 and 2 shown in Fig. 2. It follows that $n^{-2}\|\mathbf{R}\|^{2}$ is a mapping of $\mathfrak{R}$ onto a circle sector (see Fig. 2). The channel correlation matrix $\mathbf{R}$ is represented by the vector $\bar{R}$ such that

$$
|\bar{R}|=n^{-1}\|\mathbf{R}\| ; \text { angle }\{\bar{R}\}=\tan ^{-1}\{\|\mathbf{P}\| /\|\mathbf{K}\|\}
$$

Following Corollary 1, the asymptotic outage capacity is affected by the length of $\bar{R}$ but not by its angle. Consider two channels with correlation matrices represented by the vectors $\bar{R}_{1}$ and $\bar{R}_{2}$ such that $\left|\bar{R}_{1}\right|=\left|\bar{R}_{2}\right|=|\bar{R}|$ (see Fig. 2). Following Definitions 1 and 2, the channel with $\bar{R}_{1}$ is more correlated that one with $\bar{R}_{2}$. In contrast, the channel with $\bar{R}_{2}$ has more power imbalance across antennas. Nonetheless, the outage capacity of both channels is same. Therefore, the power imbalance and correlation between antennas have the same impact on the asymptotic capacity distribution of a single-keyhole channel if $\left|\bar{R}_{1}\right|=\left|\bar{R}_{2}\right|$.

To strengthen the general discussion above and to show explicitly the impact of correlation on the asymptotic outage capacity distribution, we consider, as an example, the exponential correlation matrix models for $\mathbf{R}$ [9]. In this model, the elements of correlation matrix $\mathbf{R}$ (either $\mathbf{R}_{t}$ or $\mathbf{R}_{r}$ ) are represented through a single complex correlation parameter $r$ as following

$$
R_{k m}=\left\{\begin{array}{l}
r^{m-k} ; m \geq k \\
r^{k-m} ; m<k
\end{array},|r|<1\right.
$$

where $\bar{r}$ is the complex conjugate of $r$. From (6), $n^{-1}\|\mathbf{K}\|=n^{-1}\|\mathbf{R}\|$ and $n^{-1}\|\mathbf{P}\|=0$, i.e. this model does not capture the effect of power imbalance but the correlation only. Using the properties of geometrical series, it is straightforward to show that as $n \rightarrow \infty$,

$$
n^{-2}\|\mathbf{K}\|^{2} \rightarrow \frac{1}{n} \cdot \frac{1+|r|^{2}}{1-|r|^{2}} ;|r|<1
$$

Thus, following Theorem 1, when both $\mathbf{R}_{t}$ and $\mathbf{R}_{r}$ are described by the exponential model, the asymptotic capacity distribution of the corresponding single-keyhole channel is Gaussian, since $n^{-1}$ trace $\{\mathbf{R}\}=1$ and $n^{-2}\|\mathbf{R}\|^{2} \rightarrow 0$ as $n \rightarrow \infty$. From (14), the measure of correlation increases 
monotonically with $|r|$. This fact supports Definition 1 . Moreover, $n^{-2}\|\mathbf{K}\|^{2}$ monotonically decreases with $n$, i.e. in the exponential correlation model, an increase in the number of antennas decreases $\sigma^{2}$ monotonically. Hence, the asymptotic capacity of a single-keyhole channel with exponential correlation increases with $n$ and decreases with $|r|$ at outage probabilities lower that 0.5 . The opposite is true for the capacity at outage probabilities higher than 0.5 .

\section{Multi-Keyhole Channel}

Following [6], the ideal single-keyhole channel is not often encountered in practice since the assumption of a single non-zero eigenmode is only a rough approximation for real propagation scenarios. More often, the channel may have a number of keyholes. By extending (1), the channel transfer matrix of such a multi-keyhole channel can be represented as:

$$
\mathbf{H}=\sum_{k=1}^{M} a_{k} \mathbf{h}_{r k} \mathbf{h}_{t k}^{H}=\mathbf{H}_{r} \mathbf{A} \mathbf{H}_{t}^{H}
$$

where $M$ is a number of keyholes, $a_{k}$ is the complex gain of the $k$-th keyhole, $\mathbf{h}_{t k}\left[n_{t} \times 1\right]$ and $\mathbf{h}_{r k}\left[n_{r} \times 1\right]$ are random vectors representing the complex gains from the transmit antennas to the $k$-th keyhole and from the $k$-th keyhole to the receive antennas respectively; $\mathbf{H}_{t}=\left[\mathbf{h}_{t 1} . . \mathbf{h}_{t M}\right]$, $\mathbf{H}_{r}=\left[\mathbf{h}_{r 1} . \mathbf{h}_{r M}\right]$ are $\left[n_{t} \times M\right]$ and $\left[n_{r} \times M\right]$ matrices respectively, and $\mathbf{A}$ is a $[M \times M]$ diagonal matrix with elements $\mathbf{A}_{k k}=a_{k}, k=1 . . M$.

Similarly to the single-keyhole channel, assume that for every $k, \mathbf{h}_{t k}$ and $\mathbf{h}_{r k}$ are mutually independent complex circular symmetric Gaussian vectors with corresponding correlation matrices $\mathbf{R}_{t k}=E\left\{\mathbf{h}_{t k} \mathbf{h}_{t k}^{H}\right\}$ and $\mathbf{R}_{r k}=E\left\{\mathbf{h}_{r k} \mathbf{h}_{r k}^{H}\right\}$. Suppose also, that the keyholes are independent of each other, i.e $E\left\{\mathbf{h}_{t k} \mathbf{h}_{t m}^{H}\right\}=E\left\{\mathbf{h}_{r k} \mathbf{h}_{r m}^{H}\right\}=\mathbf{0}$ for any $k \neq m$. For comparison purposes, $\mathbf{H}$ is normalized so that $E\left\{\|\mathbf{H}\|^{2}\right\}=n_{t} n_{r}$ and for every $k, \quad n_{t}^{-1} E\left\{\left\|\mathbf{h}_{t k}\right\|^{2}\right\}=1$, $n_{r}^{-1} E\left\{\left\|\mathbf{h}_{r k}\right\|^{2}\right\}=1$, which implies

$$
\sum_{k=1}^{M}\left|a_{k}\right|^{2}=1
$$

i.e. the average SNR per Rx antenna is constant regardless of the number of keyholes. Substituting (15) in (2), the instantaneous capacity of a frequency flat quasi-static multikeyhole MIMO channel with the CSI available at the Rx end only may be expressed in natural units [nat] as

$$
C=\ln \left(\operatorname{det}\left[\mathbf{I}+\gamma_{0} n_{r} \mathbf{B}_{r} \mathbf{A} \mathbf{B}_{t} \mathbf{A}^{H}\right]\right)
$$

where $\mathbf{B}_{t}=\mathbf{H}_{t}^{H} \mathbf{H}_{t} / n_{t}$ and $\mathbf{B}_{r}=\mathbf{H}_{r}^{H} \mathbf{H}_{r} / n_{r}$. Below, we consider two types of the multi-keyhole MIMO channels:

Full-Rank Multi-Keyhole Channel $\left(M \geq \min \left\{n_{t}, n_{r}\right\}\right)$ :

Theorem 2: A full-rank multi-keyhole channel is asymptotically Rayleigh fading as $M \rightarrow \infty$ if

$$
\lim _{M \rightarrow \infty} \max _{k}\left\{\left|a_{k}\right|^{2}\right\}=0,
$$

i.e. the power contribution of each single keyhole approaches zero as $M$ goes to infinity. For example, a multi-keyhole channel with equal $\left|a_{k}\right|=\sqrt{1 / M}, k=1 . . M$ satisfies (18) simultaneously keeping the adopted normalization (16). A



Fig. 3. Outage capacity distribution of $2 \times 2$ full-rank multikeyhole channel vs. the number of keyholes $M$.

proof of Theorem 2 follows directly from the LindebergFeller Theorem [10], since under the assumption of keyhole independence, each element of $\mathbf{H}$ is a sum of $M$ independent random variables. Fig 3 compares the outage capacity distributions of a $2 \times 2$ multi-keyhole channel with $\left|a_{k}\right|=\sqrt{1 / M}$ and of the equivalent Rayleigh channel. The correlation at both Tx and Rx ends and for all $M$ keyholes is represented by the exponential model [9] with $|r|=0.5$. The Kronecker model [7] was used to simulate the correlation at Tx and Rx ends in the Rayleigh channel. Clearly, the outage probability of the multi-keyhole channel decreases with $M$ and becomes close to that of the equivalent Rayleigh channel already for $M=10$.

Moreover, it can be shown that under the conditions of Theorem 2, if

$$
\mathbf{R}_{t}=\mathbf{R}_{t k}=\mathbf{R}_{t m} ; \mathbf{R}_{r}=\mathbf{R}_{r k}=\mathbf{R}_{r m} \forall k, m,
$$

the multi-keyhole channel matrix obeys the Kronecker correlation model [7]. This model significantly simplifies analytical analysis and simulation of correlated MIMO channels by allowing independent modeling of correlation at the Tx and Rx ends. The first experimental validation of the Kronecker model was given in [7]. Considering a Rayleigh channel as a limiting case of the full-rank multi-keyhole channel with $M \rightarrow \infty$ not only stresses out the relationship between the two channels but also provides a motivation for the Kronecker model.

Rank-Deficient Multi-Keyhole Channel $\left(M<\min \left\{n_{t}, n_{r}\right\}\right)$ :

Theorem 3: Let $C$ be an instantaneous capacity of the multi-keyhole channel (17). If for every $k, m=1 . . M$, $n_{t}^{-1}$ trace $\left\{\mathbf{R}_{t k}\right\}<\infty, n_{t}^{-2}$ trace $\left[\mathbf{R}_{t k}^{H} \mathbf{R}_{t m}\right] \rightarrow 0$ as $n_{t} \rightarrow \infty$, and $n_{r}^{-1}$ trace $\left\{\mathbf{R}_{r k}\right\}<\infty, n_{r}^{-2}$ trace $\left[\mathbf{R}_{r k}^{H} \mathbf{R}_{r m}\right] \rightarrow 0$ as $n_{r} \rightarrow \infty$, the following holds true as both $n_{t}$ and $n_{r}$ go to infinity:

$$
C \stackrel{p}{\rightarrow} \sum_{k=1}^{M} \ln \left(1+\left|a_{k}\right|^{2} \gamma_{0}\left\|\mathbf{h}_{t k}\right\|^{2}\left\|\mathbf{h}_{r k}\right\|^{2} / n_{t}\right)
$$

where $\stackrel{p}{\rightarrow}$ means convergence in probability. Hence, the asymptotic instantaneous capacity of a rank-deficient multikeyhole channel is the sum of the capacities of the equivalent single-keyhole channels. A proof is omitted due to the page limit. Note that the conditions of Theorem 1 for the single keyhole follow from those of Theorem 3; therefore the terms 
of the sum (20) are Gaussian. Moreover, they are independent as the keyholes are assumed to be independent. Therefore, similarly to the single-keyhole channel, the asymptotic instantaneous capacity of the multi-keyhole channel is also Gaussian with the mean $\mu$ and the variance $\sigma^{2}$ given as follows:

$$
\begin{gathered}
\mu=\sum_{k=1}^{M} \ln \left(1+\left|a_{k}\right|^{2} \gamma_{0} n_{r}\right) \\
\sigma^{2}=n_{t}^{-2} \sum_{k=1}^{M}\left\|\mathbf{R}_{t k}\right\|^{2}+n_{r}^{-2} \sum_{k=1}^{M}\left\|\mathbf{R}_{r k}\right\|^{2}
\end{gathered}
$$

Let $|\bar{R}|^{2}=n^{-2} \sum_{k=1}^{M}\left\|\mathbf{R}_{k}\right\|^{2}$, where the vector $\bar{R}$ represents the total effect of correlation and power imbalance at either Tx or Rx end. From (7),

$$
|\bar{R}|^{2}=n^{-2} \sum_{k=1}^{M}\left\|\mathbf{K}_{k}\right\|^{2}+n^{-2} \sum_{k=1}^{M}\left\|\mathbf{P}_{k}\right\|^{2}
$$

where $\mathbf{K}_{k}$ and $\mathbf{P}_{k}$ are the correlation and the power imbalance respectively created by the $k$-th keyhole. Thus, the length of $\bar{R}$ (which affects the outage capacity) is a simple summation of correlation and power imbalance measures of all $M$ keyholes. Due to this relationship, the impact of correlation and power imbalance on the asymptotic outage capacity distribution of the single-keyhole channel and of the rank-deficient multi-keyhole channel is similar; the capacity at outage probabilities less than 0.5 decreases with correlation or/and power imbalance across the antennas, where the scalar measures for correlation and power imbalance are given by Definitions 1 and 2 for each $\mathbf{R}_{k}$ respectively.

Using the Jensen inequality and the normalization in (16), it is straightforward to show that

$$
\mu \leq M \ln \left(1+\gamma_{0} n_{r} / M\right)
$$

with the equality if $\left|a_{k}\right|=\sqrt{1 / M}, k=1 . . M$. Moreover, since $M \ln \left(1+\gamma_{0} n_{r} / M\right)$ increases monotonically with $M$, the channel with more equal-gain keyholes has higher mean capacity. However, this is not necessarily true for the outage capacity. Since an increase in $M$ increases not only $\mu$ but also $\sigma^{2}$ (see (21)), the outage capacity for some outage probabilities may increase, while for others it may decrease. To demonstrate this, consider a marginal case where the channel has $M$ equal-gain keyholes and $M$ is large. From (23) $\mu \rightarrow \gamma_{0} n_{r}$ as $M \rightarrow \infty$, i.e. asymptotically does not depend on the number of keyholes. In contrast, $\sigma^{2}$ increases with $M$. Following the previous analysis for two Gaussian CDFs with the same mean and different variances, one may conclude that an increase in $M$ decreases the outage capacity of such multi-keyhole channel at outage probabilities less than 0.5 and increases it at outage probabilities higher than 0.5 . We stress that this conclusion holds true under the normalization (16) and may change if other normalization is adopted.

\section{CONCLUSION}

The multi-keyhole channel model establishes a link and emphasizes a relationship between the keyhole and Rayleigh MIMO channels. The asymptotic outage capacity of the keyhole channels (with either single or multiple keyholes) decreases with correlation or/and power imbalance at low outage probabilities. The fact that the outage capacity distribution of all considered channels is asymptotically Gaussian may indicate that the Gaussian distribution has a certain degree of universality in the outage capacity analysis of MIMO channels.

A profound reason to study the keyhole channels is not that much in practical applications; but rather because this channel is so much different from the Rayleigh one (which was extensively studied and is well understood by now). The investigation of the keyhole channels can reveal how well a system performs in channels other than the Rayleigh ones, how much the results established for the Rayleigh channels apply elsewhere (i.e. robustness), and whether new techniques need to be developed. As a byproduct of this study, a new (scalar) measure of correlation and power imbalance is introduced. This measure allows complete rather than partial ordering of the channels (contrary to the ordering based on the majorization theory, which allows only a partial ordering), and can be applied to other channels, whose capacity depends on the norm of correlation matrices, for example, to correlated Rayleigh channels [11].

\section{REFERENCES}

[1] G. J. Foschini and M. J. Gans, "On Limits of Wireless Communications in a Fading Environment when Using Multiple Antennas", Wireless Personal Commun, vol. 6, no. 3, pp. 311-335, March 1998.

[2] M. Chiani, M. Z. Win and A. Zanella, "On the Capacity of Spatially Correlated MIMO Rayleigh-Fading Channels", IEEE Trans. on Information Theory, vol. 49, no. 10, pp. 2363-2371, Oct. 2003.

[3] D. Chizhik, G. J. Foschini and R. A. Valenzuela, "Capacities of Multi-Element Transmit and Receive Antennas: Correlations and Keyholes", Electronics Letters, vol. 36, no. 13, pp. 1099-1100, June 2000.

[4] G. Levin and S. Loyka, "Capacity Distribution of a Correlated Keyhole Channel", in Proc. Canadian Workshop on Information Theory CWIT 2005, pp. 319-322, Montreal, QC, June 2005.

[5] H. Shin and J. H. Lee, "Capacity of Multiple-Antenna Fading Channels: Spatial Fading Correlation, Double Scattering, and Keyhole", IEEE Trans. on Information Theory, vol. 49, no. 10, pp. 2636-2646, Oct. 2003.

[6] P. Almers, F. Tufvensson and A. F. Molisch, "Measurements of Keyhole Effect in a Wireless Multiple-Input Multiple-Output (MIMO) Channel", IEEE Communications Letters, vol. 7, no. 8, pp. 373-375, Aug. 2003.

[7] J. P. Kermoal, L. Schumacher, K. I. Pedersen, P.E. Mogensen and F. Frederiksen, "A Stochastic MIMO Radio Channel Model with Experimental Validation", IEEE J. Sel. Areas Commun., vol. 20, no. 6, pp. 1211-1226, Aug. 2002.

[8] H. Bosche and E. A. Jorswieck, "On the Ergodic Capacity as a Function of the Correlation Properties in Systems with Multiple Transmit Antennas without CSI at the Transmitter", IEEE Trans. on Commun., vol. 52, no. 10, pp. 1654-1657, Oct. 2004.

[9] S. Loyka, "Channel Capacity of MIMO Architecture Using the Exponential Correlation Matrix", IEEE Communication Letters, vol. 5, no. 9, pp. 369-371, Sep. 2001.

[10] M. Fitz, Probability Theory and Mathematical Statistics, John Willey \& Son, Inc., New-York, London, $3^{\text {rd }}$ Ed., 1963.

[11] C. Martin and B. Ottersten, "Asymptotic Eigenvalue Distributions and Capacity for MIMO Channels under Correlated Fading", IEEE Trans. On Wireless Commun, vol. 3, no. 4, pp. 1350-1358, July 2004. 